\title{
The Mechanics of Accommodation in Relation to Presbyopia
}

\author{
R. F. FISHER
}

London

\section{Summary}

The cause of presbyopia is closely related to the force of contraction of the ciliary muscle and the resistance to deformation of the crystalline lens. Two views are currently in conflict. The view of Donders ${ }^{20}(1864)$ that presbyopia is caused by a decrease in the force of contraction of the ciliary muscle with age, and the opposing view of Helmholtz ${ }^{3}$ (1855) that the lens becomes more difficult to deform with age due to lenticular sclerosis.

The present paper shows that, in fact, the ciliary muscle undergoes a compensatory hypertrophy as accommodative amplitude decreases with age. The force of contraction is about $50 \%$ greater at the onset of presbyopia than in youth. However, because of increased lenticular resistance its effect on the amplitude of accommodation is small. It is shown that the reason the lens becomes more difficult to deform is not because of lenticular sclerosis, since the lens substance does not lose water. ${ }^{19}$ The increased difficulty of deformation is because the capsule loses its elastic force with age and the lens fibres, particularly in the nucleus, become more compacted.

It is generally accepted that relaxation of the zonule causes the lens to become more rounded and increase its refractive power. Thus any increase in accommodation caused by a change in shape of the lens is solely determined by the elastic forces released within the lens itself. The importance of the capsule in generating these forces was emphasised by Fincham ${ }^{1}$ who thought that the lens substance was merely plastic in nature. However, Weale ${ }^{2}$ suggested that there was an interplay of forces between the lens capsule and the lens substance with both structures possessing an inherent elasticity.

The present paper summarises a series of experiments performed over the years to determine the precise magnitudes of the elastic properties of lens capsule and lens substance. These experiments have made it possible to determine the effect of ageing changes both in the lens and ciliary body together with their combined role in reducing accommodation as age advances.

The importance of vitreous in accommodation Before discussing the detailed mechanics of accommodation the rôle of the vitreous is of prime importance; indeed two rival theories were developed to explain its function. In the first theory - the Helmholtz theory ${ }^{3}$ the vitreous had a passive and unimportant rôle, whereas in the second theory - the Tscherning theory ${ }^{4}$ the vitreous was all important and prevented the lens from moving backwards. In recent years this view was discounted but has been revived by Coleman. ${ }^{7}$ Fisher showed that in vitro ${ }^{5}$ and in vivo $^{6}$ the lens could accommodate perfectly well in the absence of vitreous. Recently, Coleman ${ }^{8}$ has again defended the importance of vitreous in accommodation and produced a "hydraulic suspension theory" of 
Accommodation, showing that the vitreous pressure does indeed rise as the eye accommodates but the effect is small. It Hould appear that this rise of vitreous pressure can merely damp down vibrations of the lens when accommodation has been completed (see Table 1).

Investigations which have determined the elasticity and thickness of the capsule throughout life

\section{Thickness}

The thickness of the unfixed capsule was determined at every age. During the time that accommodation was at its maximum, the capsule was thickest at the equator of the lens and did not show the peripheral thickenings ${ }^{9}$ described by Fincham. In later life however, when presbyopia was well advanced a considerable thickening occurred at the insertion of the zonules. Thus on simple anatomical grounds flattening of the lens periphery could not be due to differences in thickness of the capsule during the accommodative period of life. (Fig. 1).

\section{Elasticity}

The amount of energy which has been stored in the capsule when it passes from the unaccommodated state was of such a magnitude that it would produce a change in force of about $1.0 \mathrm{gm}$ in the entire zonule. ${ }^{10,11}$ O'Neile and Doyle ${ }^{12}$ calculated that a force of about 32 gms would be required to produce a "conical" shape to the lens as proposed by Fincham. A further confirmation of about 1.0 gms change in zonular tension during accommodation was obtained directly by stressing the intact lens by means of centrifugal force. ${ }^{13}$ Thus on mechanical grounds the flattening of the lens could not be produced by the small change of zonular force observed during accommodation.

Investigations which have determined the elasticity of the intact lens at every age

The lens was spun around its anterio-posterio polar axis to mimic the radial pull of the zonule. By altering the speed of spinning, the radial force acting on the lens could be varied. The greatest difference between polar and equatorial elasticity of the lens, with a ratio of the polar to equatorial elasticity of about 0.6 , occurs at the age of

Table I Effect of absence of vitreous on amplitude of accommodation and movements of the lens

Right eye Left eye
vitreous absent vitreous present
Mean SD Mean SD

Amplitude of accommodation

(dioptres)

$9.7 \pm 1.05 \quad 8.9 \pm 0.51$

Movement of anterior pole of lens $(\mathrm{mm})$
$0.14 \pm 0.04$
$0.15 \pm 0.04$

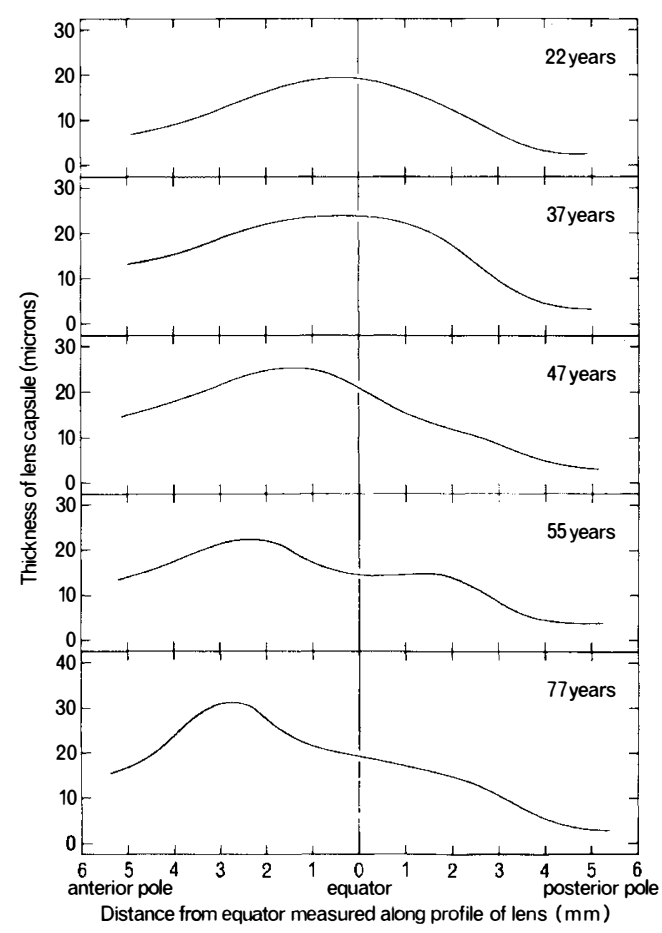

Fig. 1. Changes of capsular thickness with age in typical human lenses. 
$30 .{ }^{13}$ This is the age that Brown ${ }^{14}$ noticed the greatest "conical" shape of the lens.

From these experiments it would seem that the shape changes observed in the lens are probably due to the elastic properties of the intact lens rather than the capsule. Also the force generated by the capsule is similar to that required to deform the lens provided the movement of the zonule does not decrease with age.

Investigations which have determined the force of accommodation and the cause of presbyopia

The present method is novel in that it seeks to establish the relationship between the optical properties of the crystalline lens and the forces to which it is subjected by measuring in absolute units both quantities on the same lens. Previously, this was done indirectly by studying the relationship between accommodation and convergence or the action of drugs on the eye. In all these experiments, it was assumed that accommodative force was directly proportional to the amplitude of accommodation. The new method isolates the ciliary body zonular lens complex and determines the relationship between changes in thickness of the lens, diameter of the ciliary ring and the amplitude of accommodation.

Subsequently the lens is detached from the zonule by microdissection and then stressed radially by centrifugal forces so that the relationship between force and change in thickness of the lens could be measured. Using the change in lenticular thickness as a transfer variable common to both experiments the relationship between the accommodation power of the lens and zonular tension was determined. ${ }^{5}$

It was found that the force of contraction of the ciliary muscle was proportional to the square of the amplitude of accommodation. From the time of Hess ${ }^{15}$ it had been assumed that ciliary force was proportional to accommodation. Hoeve and Flieringa ${ }^{16}$ used this concept of proportionality to justify their introduction of the term "myodioptre" which indicated the amount of power that the ciliary muscle had in reserve and did not exert in maximal accommodation. It was calculated to be about twice as great as that actually observed in maximal accommodation. They came to this conclusion because at low amounts of convergence, and when partially paralysed by drugs, the ciliary muscle showed a great loss of activity. This does not initially occur at maximum accommodation where no loss of activity could at first be detected. These observations may now be easily explained because force is related to the square of the amplitude. Indeed from this square law the change in ciliary muscle force necessary for a given change in accommodative amplitude is proportional to the amplitude of accommodation. Thus at the region of maximum contraction a small change in force makes much less difference to the increase in amplitude of accommodation than the same change in force necessary to initiate accommodation.

It so happens that the two amplitudes of accommodation where measurements were made differed by a factor of between 2 and 3 , so by simple extrapolation it was thought that the latent power of the ciliary muscle was two to three times greater than that observed at maximum contraction but both values, of course, had to be expressed in dioptres. The cause of presbyopia was also revealed by the present experiments as it was found that although the force of the ciliary muscle increased by about $50 \%$ to that in youth the ageing changes in the lens prevented it from deforming, ${ }^{17,18,19}$ and were thus solely responsible for the decline in accommodation with age.

\section{References}

${ }^{1}$ Fincham, EF: The mechanism of accommodation. Br J Ophthalmol 1937, 21:, Monograph suppl 8.

2 Weale RA: Presbyopia. Br J Ophthalmol 1962, 46: $660-8$.

${ }^{3}$ Helmholtz $\mathrm{H}$ : Über die Akkommodation des Auges. Albrecht v Graefes Arch Ophthalmol 1855, 1: 1-74.

${ }^{4}$ Tscherning M: Le méchanisme de l'accommodation. Ann Oculist Paris 1904 131: $168-79$. 
${ }^{5}$ Fisher RF: The force of contraction of the human ciliary muscle during accommodation. J Physiol 1977, 270: 51-74.

${ }^{6}$ Fisher RF: Is the vitreous necessary for accommodation in man. Brit J Ophthalmol 1983, 67: 206.

${ }^{7}$ Coleman DJ: Unified model for accommodative mechanism. Am J Ophthalmol 1970, 69: 106379.

${ }^{8}$ Coleman DJ: On the hydraulic suspension theory of accommodation. Trans Am Ophthalmol Soc 1986, 84: 846-8.

${ }^{9}$ Fisher RF and Pettet BE: The postnatal growth of the capsule of the human crystalline lens. $J$ Anat 1972 112: 207-14.

${ }^{10}$ Fisher RF: Elastic constants of lens capsule. $J$ Physiol 1969, 201: 1-19.

${ }^{11}$ Fisher RF: The significance of the shape of the lens and energy changes in accommodation. $J$ Physiol 1969, 201: 21-47.

12 O'Neill and Doyle JM: A thin shell analysis of the human lens. Vision Res 1968, 8: 193-206.
${ }^{13}$ Fisher RF: The ciliary body in accommodation. Trans Ophthalmol Soc UK 1986, 105: 208-17.

${ }^{14}$ Brown, N: The changes in shape and internal form of the lens of the eye on accommodation. Exp Eye Res 1973, 15: 44159.

${ }^{15}$ Hess C: Beobuchtungen über den akkommodationsuorgang Klin $\mathrm{Mbl}$ Augenheilk 1904, 42: 309-15.

${ }^{16}$ Van der Hoeve and Flierenga $\mathrm{HJ}$ Accommodation. Br J Ophthalmol 1924, 8: 97-106.

${ }^{17}$ Fisher RF: The elastic constants of the human lens. J Physiol 1971, 212: 147-80.

${ }^{18}$ Fisher RF: Presbyopia and the changes with age in the human crystalline lens. J Physiol 1973, 228: 765-79.

${ }^{19}$ Fisher RF and Pettett BE: Presbyopia and the water content of the human crystalline lens. $J$ Physiol 1973, 234: 443-7.

${ }^{20}$ Donders FC: On the anomalies of accommodation and refraction of the eye. London: New Sydenham Soc (1864). 